\title{
Environmental Monitoring of Electric Power Transmission Corridor Based on Satellite Remote Sensing
}

\author{
Yang zhi $^{12+}$, Ou wenhao ${ }^{1,2}$, Wei liguang ${ }^{3}$, Fei xiangze ${ }^{1,2}$, Li chuang $^{1,2}$, Zhao binbin $^{1,2}$, Ma xiao $^{1,2}$ \\ ${ }^{1}$ State Grid Corporation of China, Beijing 100031, China \\ ${ }^{2}$ China Electric Power Research Institute, Beijing 100192, China \\ ${ }^{3}$ Beijing Piesat Information Technology Co. Ltd Beijing 100195 , China
}

\begin{abstract}
With the rapid development of national economy, the current power industry and power technology have made great progress in recent years. It is of vital importance to ensure the safety of electric power transmission corridors and related facilities. At present, the inspection of electric power transmission corridors mainly relies on manpower in China, which is not only of great labor intensity, high labor cost and low efficiency. Therefore, this paper proposes a method of surveying typical environmental features of electric power transmission corridor based on high-resolution remote sensing image. At first, the normalized differential vegetation index (NDVI) and normalized difference water index (NDWI) time series were constructed using multi-period remote sensing images in the research area. Then harmonic analysis is applied to the indexes time series to obtain the harmonic characteristics. Finally, support vector machine (SVM) was used to classify five typical environmental features including artificial active area, water, bare land, farmland and forest, The overall classification accuracy is $90.29 \%$, which is better than the result only using indexes series for classification.
\end{abstract}

Keywords: Electric Power Transmission Corridors; Ground object inspection; Remote sensing image; Index time series; Harmonic analysis

\section{Introduction}

Transmission lines are the lifeblood of the national economy and the lifeline of people's livelihood [1]. The safety and stability of the line and its related facilities is of vital importance. In recent years, the national economy has developed rapidly, and the illegal buildings in the line protection area are more serious. It results in that the vertical or horizontal distance of the transmission line and the house is less than the safe distance; In addition, there are some other behaviors that leave hidden dangers for the safety of power line [2-5], such as the explosion of the mountain near the overhead transmission channel, causing the wire to be interrupted, planting trees artificially in the line protection area, etc. The accidents of transmission line are increasing due to the above-mentioned reasons, so the need to strengthen the inspection of the surrounding environment of the transmission line is becoming more and more urgent [6]. In order to realize the normal and safe operation of the power line, the power department needs to conduct regular inspections on the transmission line and its surrounding objects, that is, to monitor the ground objects around the transmission channel. At present, the inspection work of transmission lines in China mainly relies on manual inspection, which not only has high labor intensity, difficult working conditions, but also low efficiency [7-8]. At the same time, there are certain defects in the scope of monitoring, timeliness and reliability [9]. Therefore, a kind of fast, large-scale, real-time technology of line monitoring is needed.

Remote sensing is a kind of detection technology that emerged in the 1960s. Based on the theory of electromagnetic wave transmission, the probes are used to detect and collect electromagnetic wave (radiation)

+ Corresponding author. Tel.: 010-82812786-833.

E-mail address: yangzhi0713@foxmail.com. 
information on earth surface. After the transmission, processing, analysis and classification of information, the classification and recognition of various types of features are realized [10]. Remote sensing image information has the advantages of real-time, fast and low cost and remote sensing data is generated according to certain rules and analysis. It is subjectively less influential and can reflect the essential characteristics of the original features. So it has certain objectivity [11].

The application of remote sensing in electric power can be traced back to the last century. From the 1950s onwards, helicopters were used to conduct line inspection in foreign countries [12]. Most of the current research in China is focused on the use of UAV for line inspection [13-15]. Compared with UAVs and aerial remote sensing of line inspection, satellite observations have unparalleled advantages, such as wide coverage, fast access to information, short update period, fewer limited conditions of getting information, large amount of information acquired, and many sensing methods. It is suitable for monitoring areas which has harsh geographical conditions and natural environment that is difficult to reach [16]. Therefore, satellite observation is more suitable for monitoring of a wide range and long-distance transmission channel environments.

Due to the diversity of features, image noise, terrain and other factors, there are obvious spectral overlaps between different features in remote sensing images, which are prone to "homologous" and "foreign matter" phenomena [17]. Classification using single-period remote sensing images makes it difficult to meet the spatial and temporal heterogeneity of classification, so it is difficult to obtain high classification accuracy in practical applications [18-19]. Compared with the single-period image, the information of the multi-period time-series image can better compensate the misclassification caused by the insufficient resolution of the single-period image and improve the classification accuracy [20]. For multi-period time-series image, it is very important to mine the information and determine the characteristics of different features to improve the classification accuracy.

The harmonic analysis method is not only used for signal processing analysis, but also for multi-period time-series image processing, that is , Harmonic analysis of time-series (HANTS) proposed by Jakubauskas,M.E. Etc. [21].

Harmonic analysis of time-series can not only accurately describe the change information in time series images [22], but also quantitatively express the dynamics of various types of ground objects [23].

Therefore, this paper takes Yongning County as the research area and uses the GF-1 remote sensing image to establish multi-period time-series remote sensing images of the study area. Then harmonic analysis is performed on the basis of indices such as NDVI and NDWI. Finally, the support vector machine is used to classify the features in the study area.

The type of ground objects has an important influence on the inspection of transmission lines. Artificial activity areas and farmland are high-risk areas for human activities, and it is easy to cause damage to transmission lines. For example, in the construction of roads and bridges and construction sites, large-scale construction equipment is constructed in the transmission line protection area, causing damage to the transmission line and its ancillary facilities. When the transmission line passes through the farmland, the plastic film used in the agricultural greenhouse is not fixed firmly and is short-circuited by contact with the electric wire. Planting high pole plants can destroy electrical facilities. In the forest area, the distance between the transmission line and the branches is too close, which may cause potential safety hazards. The construction of water conservancy and adjacent facilities along the river will have an impact on the transmission lines along rivers and lakes. Therefore, in accordance with the "GB50545-2010 110kV-750kV overhead transmission line design specifications", "GB 50548-2010 330kV - 750kV overhead transmission line survey specifications", "GB 50665-2011 1000KV overhead transmission line design specifications" and other transmission line specifications, the ground objects are classified into five categories: artificial activity areas, water bodies, bare land, farmland, and forests. The classification results can provide information support for a wide range of long-distance power line monitoring and facility construction.

\section{Research Area Overview and Data}

\subsection{Research area overview}


Yongning County is affiliated to Yinchuan City, which is located in the middle of the Yellow River Irrigation District of Yinchuan Plain. It is connected to the Yellow River in the east and Helan Mountain in the west, with a total area of about 934 square kilometers. Yongning County is a mid-temperate arid climate zone with obvious continental climate characteristics. The terrain of Yongning County is high in the west and low in the east. It is inclined to the southeast to the northeast. The whole territory can be divided into five geomorphic units, namely: Helan Mountain, Alluvial fan, River Old Terrace, Windy Land, and The alluvial plains of the Yellow River. The Helan Mountain is mainly located in the northwest of the county; The alluvial fan is a fan-sloping plain formed by the alluvial deposits of the Helan Mountains. It is mainly a pastoral area now. The surface dunes of the windy sands are undulating, and some are Pingsha, mainly the Guolin New District. The alluvial plains of the Yellow River mainly include lake swamps caused by the diversion and siltation of the Yellow River.

\subsection{Distribution of features}

The cultivated land in Yongning County accounts for about 45\% of the total area of Yongning County, mainly distributed in the old irrigation area of the lower reaches of the Yellow River. The cultivated land accounts for $80 \%$ of the total cultivated land in the county [24], and the typical crops are mainly wheat, corn and rice. Helan Mountain on the western boundary of Yongning County is the dividing line between the Chinese and foreign water systems, and all of them belong to the Yellow River water system. The west comes from the small ditch of the Honggou Mountain in the Helan Mountain to the alluvial fan and the old terrace. In addition to the Huanghe Mountain Flood Ditch, there are mature irrigation canals and drainage facilities and numerous lake swamps on the eastern plain. The main tree species in Yongning County are Pinus tabulaeformis, spruce, and aspen, and also include peach trees, apricot trees and other fruit trees, all of which belong to evergreen trees.

\subsection{Data collection}

The GF-1 satellite is the first satellite of China's high-resolution Earth observation system. It was launched into orbit by the Long March II carrier rocket on April 26, 2013. The GF-1 is equipped with two 2meter spatial resolution full-color/8-meter spatial resolution multi-spectral cameras and four multi-spectral wide-format cameras with 16-meter spatial resolution. This paper selects $8 \mathrm{~m}$ resolution multi-spectral image and $2 \mathrm{~m}$ full-color image. In order to improve the stability of the model, a total of 17 periods of remote sensing images were selected from October 1, 2015 to November 27, 2016.

\section{Data Processing}

\subsection{Data preprocessing}

Before the data is analyzed and processed, pre-processing such as radiation correction is needed to eliminate the influence of sensor factors on the spectral information of the ground object.

Proceed as follows:

1) We perform radiometric calibration and atmospheric correction on images.

2) We use the RPC (rational polynomial coefficient) file and the ground control point to perform geometric correction on each original image.

3 ) The fusion of $8 \mathrm{~m}$ spatial resolution and $2 \mathrm{~m}$ full-color image is conducted.

4) The fused image is clipped and chronologically arranged to obtain the 17-period time series image of the study area.

\subsection{Normalized index model}

The basic principle of the ratio index structure is to find the two bands with the largest difference in the features to be studied in all bands, and then increase the difference between the two by the ratio operation. The information of the features of interest is enhanced on the index image, while the other features are suppressed to achieve the purpose of distinguishing the features [25]. The ratio index is often normalized to normalize its range of values to $[-1,+1]$. In this paper, considering the distinguishing features of the study area and the characteristics of high-resolution images, two normalized ratio-type indices are selected for the 
extraction of typical features. They are the Normalized Vegetation Index (NDVI) and the Normalized Difference Water Index (NDWI) [26].

\subsection{Harmonic analysis}

Harmonic analysis refers to the non-sinusoidal periodic signal is spread into a series of harmonics according to the Fourier series to obtain the amplitude and phase information of each harmonic in the signal.

The core idea is to use the least squares method and the Fourier transform to successively fit the changes of the NDVI curve over the entire period.

Non-sinusoidal waves contain a large number of harmonics, and different waveforms contain different harmonic components.

Any periodic signal about time can be expanded into a Fourier series, that is, a sum of infinite sine and cosine functions.

The periodic function $\mathrm{f}(\mathrm{t})$ with a period of $\mathrm{T}$ satisfying the Dirichel condition, at successive points, can be represented by a linear combination of the following trigonometric functions (Fourier series):

$$
f(t)=\frac{A_{0}}{2}+\sum_{n=1}^{\infty}\left(a_{n} \cos (n w t)+b_{n} \sin (n w t)\right)
$$

Here, $w=2 \pi t, a_{n}=\frac{2}{T} \int_{-\frac{T}{2}}^{\frac{T}{2}} f(t) \cos (n w t) d t, b_{n}=\frac{2}{T} \int_{-\frac{T}{2}}^{\frac{T}{2}} f(t) \sin (n w t) d t, n$ is an integer, $n>=0$. Let $c_{\mathrm{n}}=\sqrt{\mathrm{a}_{\mathrm{n}}^{2}+\mathrm{b}_{\mathrm{n}}^{2}}$, then (1) can $\overline{\mathrm{B} e}$ written as:

$$
f(t)=\frac{A_{0}}{2}+\sum_{n=1}^{\infty} C_{n} \cos \left(2 n \pi t / L+\varphi_{h}\right)
$$

where is the amplitude of the nth harmonic, - is also called the amplitude of the 0th harmonic, is the initial phase of the analysis of the hth harmonic, $\mathrm{n}$ is the harmonic order, and $\mathrm{L}$ is the period. The higher harmonics are generally mixed with noise information, and the amplitude and phase respectively carry the energy and radiation peak position information of the ground wave band, reflecting the local characteristic information of the spectrum.

\section{Results and Accuracy Verification}

\subsection{Harmonic analysis of NDVI}

NDVI can reflect the growth of vegetation, and the trend of its value directly reflects the growth and change process of vegetation. Different vegetations have different NDVI and variation characteristics. Therefore, different vegetations can be distinguished by NDVI sequences. By selecting samples, the NDVI sequences of several typical features such as water body, artificial activity area, farmland 1, farmland 2, and forest are established, as shown in Figure 1. Since the area of Yongning County is dominated by evergreen trees, except for the period from November to March, the NDVI curve of the forest remains high throughout the year; The NDVI of Farmland 1 began to rise from April, peaked in June, and fell to near 0 in July. The NDVI curve of farmland was in line with the phenological cycle of spring wheat. The NDVI of farmland 2 began to rise from June, reached the peak period in July, and fell to near 0 in September. The NDVI curve characteristics of the farmland were in line with the phenological cycle of late rice/corn; The NDVI curve of the water body fluctuates around -0.4 throughout the year; The NDVI curve of the artificial activity area and the bare land fluctuated around 0 throughout the year.

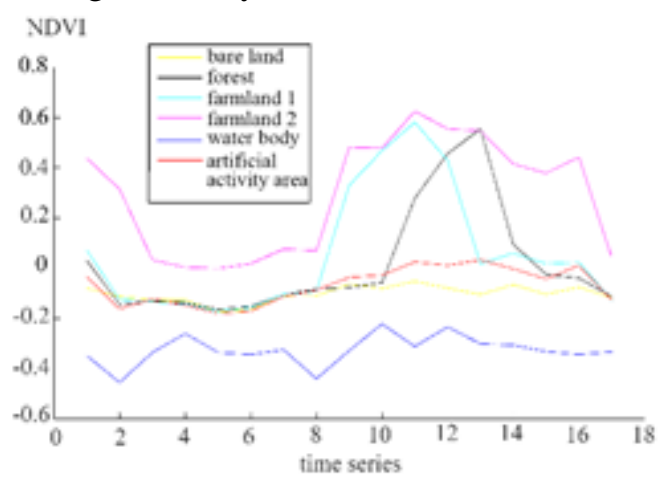

Fig. 1: The NDVI time series of typical features. 
The Nth harmonic analysis is performed on the established NDVI sequence, and the harmonic residuals are shown in Figure 2. The harmonic residuals of NDVI sequence reflect the average value of NDVI over the entire time range, to some extent indicating the overall productivity of the vegetation. Its amplitude of the 14th harmonic is shown in Figure 2. The harmonic amplitude reflects its energy. The larger the harmonic amplitude, the more obvious the periodic variation pattern reflected in the original signal. For example, the first harmonic reflects a single-peak seasonal variation pattern with a period of 12 months, and the second harmonic reflects a single-peak seasonal variation pattern with a period of 6 months.

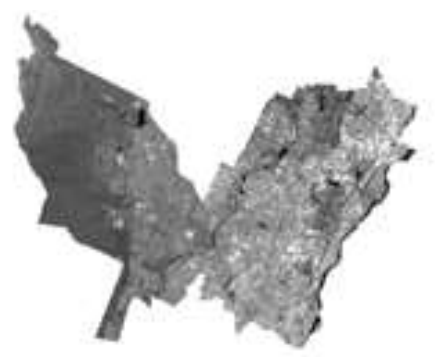

Harmonic residual of NDVI time series

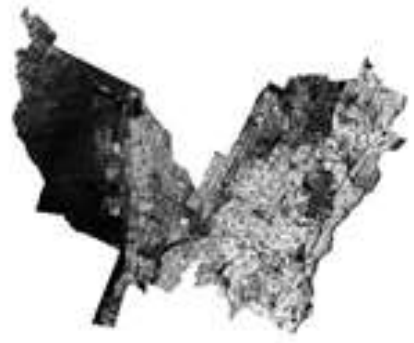

The first harmonic amplitude of NDVI time series

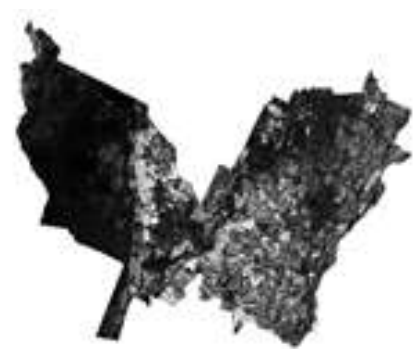

The third harmonic amplitude of NDVI time series

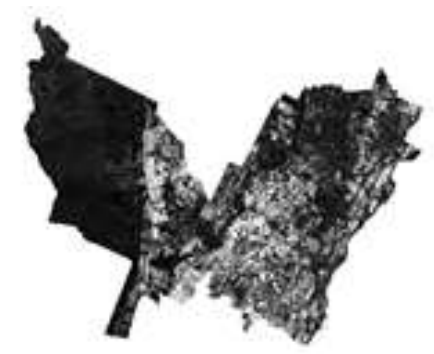

The second harmonic amplitude of NDVI time series

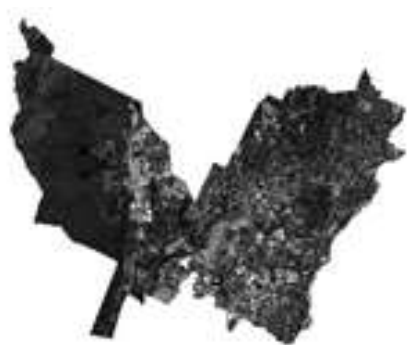

The fourth harmonic amplitude of NDVI time series

Fig. 2: Harmonic amplitude of the NDVI time series.

By selecting the training samples of typical features, the mean of the harmonic amplitudes of different features is shown in Figure 3. It can be seen that the NDVI harmonic residual value of the forest is the largest, which is because most of the forest belongs to evergreen trees. From the forest NDVI sequence, we can see that the NDVI remains high except for the period from November to March. Followed by part of the farmland, most of the farmland is cooked once a year, some are two crops a year, and vegetables with short growth cycle are generally grown in winter. So the NDVI sequence harmonic residual value of farmland is usually slightly higher than 0; The NDVI sequence of water body, building and artificial activity area has the lowest harmonic residual value, and its harmonic residual value is generally far below zero. The first harmonic amplitude of farmland and forest is large, because the obvious single-peak harmonic phase reflects the time when the harmonic peak appears. The smaller the value, the earlier the harmonic peak appears in the periodic variation mode. 


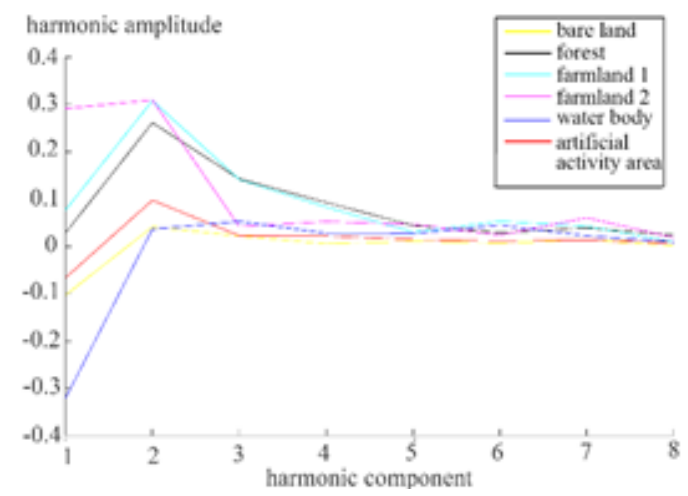

Fig. 3: Harmonic component sequence of the NDVI time series of typical features

\subsection{Harmonic analysis of NDWI}

The NDWI index is currently the most widely used water body index for water extraction. By selecting samples, the NDWI time series of different features are shown in Figure 4. The NDWI of the water body remains high throughout the year and is significantly different from other features.

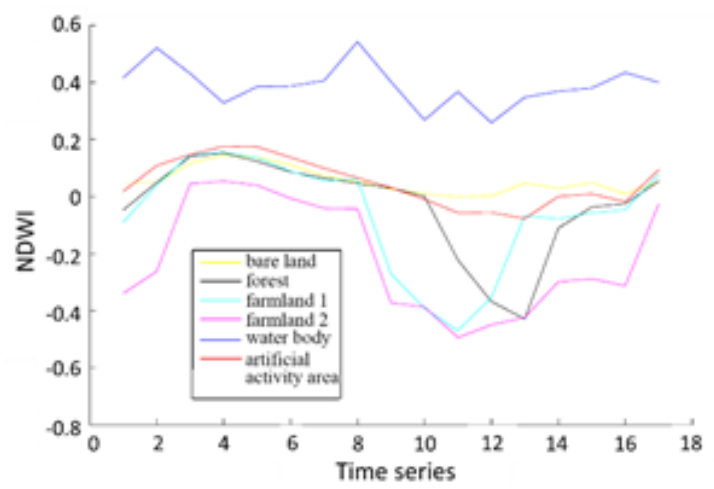

Fig. 4: The NDWI time series of typical features.

Harmonic analysis is performed on the established NDWI sequence, and the harmonic amplitudes of the harmonic residuals are shown in Fig. 5.

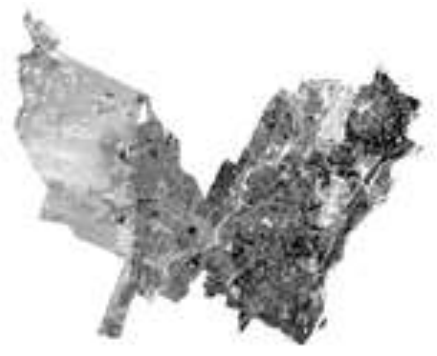

The harmonic residual of NDWI time

varies

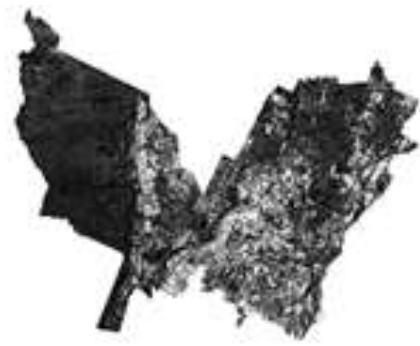

The second harmonic amplitude of NDWI time varies

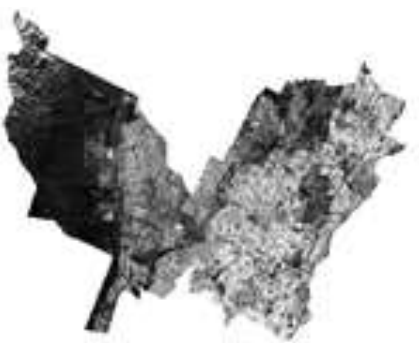

The first harmonic amplitude of NDWI time varies

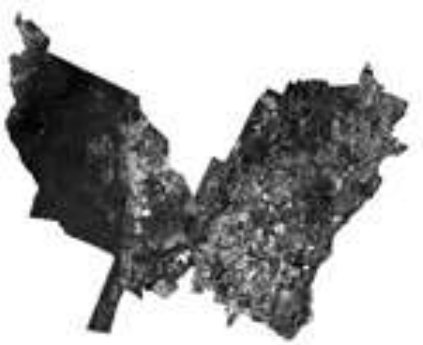

The third harmonic amplitude of NDWI time varies

Fig. 5: Harmonic amplitude of the NDWI time series. 
The amplitude of the NDWI harmonic components of various types of features is shown in Figure 6 by selecting a certain sample. Since the NDWI index of the water body remains high over the entire time range and the fluctuation is small, the harmonic residual value of the water body is high, and the first harmonic amplitude is low compared with other ground objects. The NDWI index of farmland and forest has lower harmonic residual value and higher first harmonic amplitude due to the apparent single-peak seasonal variation pattern over its entire time series. The NDWI index of the artificial residual area and the building have lower harmonic residuals, and the first harmonic amplitude is higher than 0.1 , which is due to the low amplitude of the single-peak seasonal variation over the entire time series.

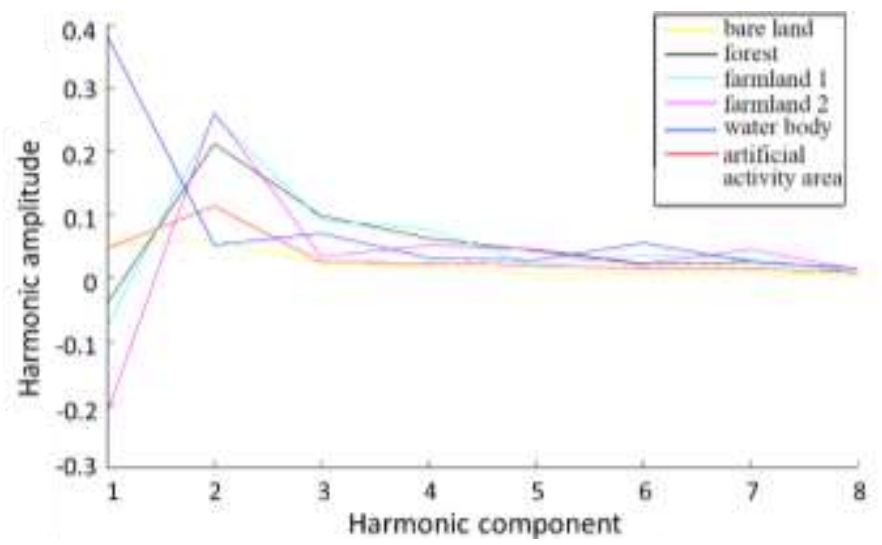

Fig. 6: Harmonic component sequence of the NDWI time series of typical features.

\subsection{Classification result}

The method of support vector machine is used to classify bare land, water body, artificial activity area, farmland and forest by selecting the critical period image of vegetation, namely March, April, September, October and NDVI harmonic residuals, first and second harmonic amplitude, NDWI harmonic residual, first and second harmonic amplitude as characteristic bands .The classification result is shown in Fig. 7.

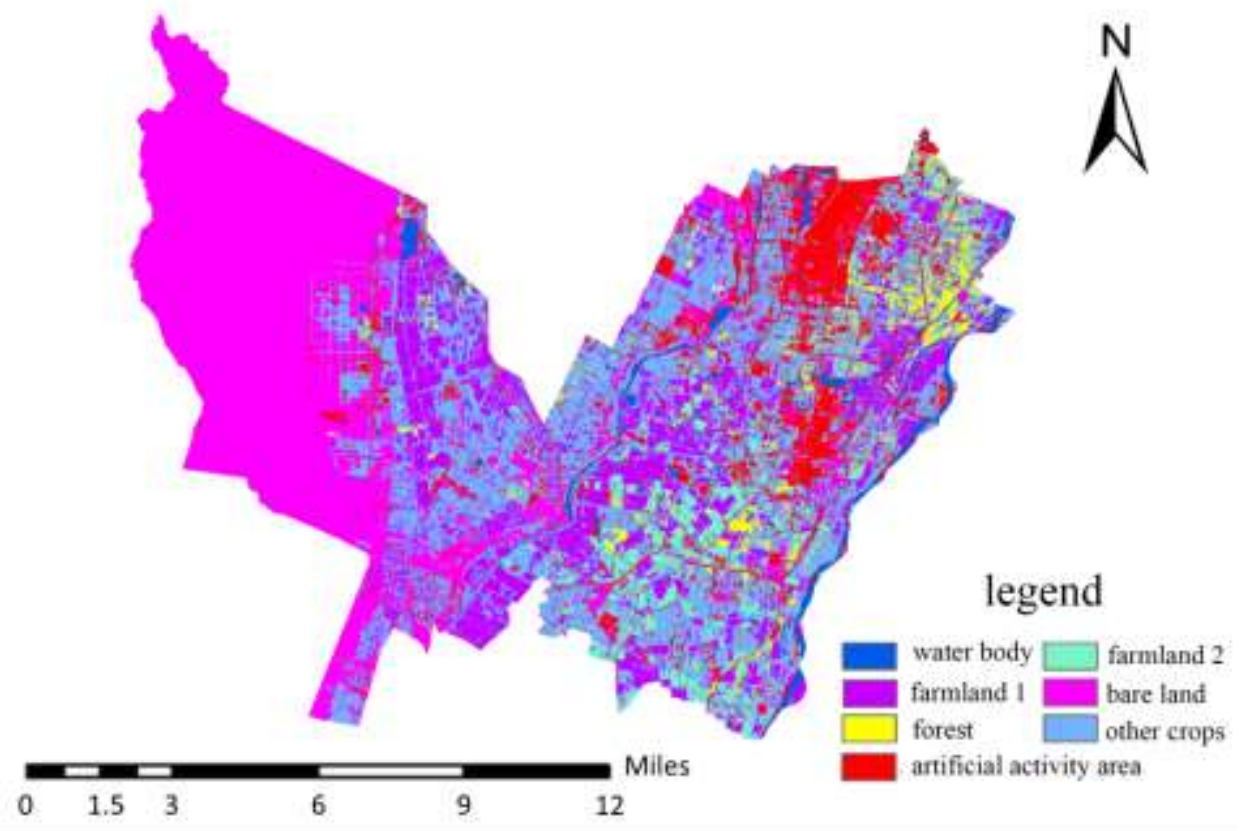

Fig. 7: Classification results.

\subsection{Precision verification}

The final classification accuracy is evaluated by selecting 100 verification points in each type of land by field sample and visual inspection. The farmland is divided into farmland 1, farmland 2 and other crops. Therefore, 300 checkpoints are selected and merged in the confusion matrix. The confusion matrix for the classification results is shown in Table 1. The drawing accuracy of water bodies, artificial activity areas, forests, farmland, and bare land is $90.00 \%, 80.00 \%, 90.00 \%, 95.67 \%$, and $85.00 \%$, respectively. The user 
accuracy is $100 \%, 76.92 \%, 95.74 \%, 89.97 \%, 91.40 \%$, and the overall accuracy is $90.29 \%$. The overall accuracy of classification using NDVI sequences directly is $83.46 \%$.

Table 1: Confusion matrix

\begin{tabular}{ccccccc}
\hline Land type & Water body & Artificial activity areas & Forests & Farmland & Bare land & The user accuracy \\
\hline Water body & 90 & 0 & 0 & 0 & 0 & $100.00 \%$ \\
Artificial activity areas & 5 & 80 & 0 & 11 & 8 & $76.92 \%$ \\
Forests & 0 & 3 & 90 & 1 & 0 & $95.74 \%$ \\
Farmland & 5 & 10 & 10 & 287 & 7 & $89.97 \%$ \\
Bare land & 0 & 7 & 0 & 1 & 85 & $91.40 \%$ \\
The drawing accuracy & $90.00 \%$ & $80.00 \%$ & $90.00 \%$ & $95.67 \%$ & $85.00 \%$ & $90.29 \%$ \\
\hline
\end{tabular}

\section{Conclusion}

There are huge differences in the peaks and troughs of the exponential time series of different features. In this paper, by using the GF-1 image time series, harmonic analysis of the established time series of the NDWI and NDVI is used to extract effective feature features, namely harmonic residuals and harmonic amplitudes. With the SVM classifier, the overall classification accuracy of the final classification combined with the field sample is $90.29 \%$, which is more accurate than the NDVI time series alone. The NDVI and NDWI index sequences were added to the classification, and the vegetation and water body recognition effects were better. The harmonic amplitude and residuals of remote sensing image time series are sensitive to different land types, and have the advantages of convenient access, timely data update and wide range. This shows that remote sensing recognition based on harmonic analysis is feasible for typical ground inspections in power channel environments.

In this paper, a total of 17 images of the time series from October 1, 2015 to November 2016 were selected. However, due to the limitation of the time series resolution, the harmonic phase thus obtained is still not representative, and it is not enough to accurately describe the phenological information, so the harmonic phase is not used for classification in the classification. Therefore, the research methods and results can further improve the temporal resolution of the image and better use the harmonic phase information to improve the classification accuracy.

\section{Acknowledgements}

This work was supported by a grant from National Key R\&D Program of China (2016YFB0502504, 2016YFB0502500), the science and technology project of State Grid (Research and application of threedimensional inspection technology for overhead transmission lines in sichuan-tibet and tibetan-china interconnection projects, 5442GY180025) and Academy Innovation Fund of China Electric Power Institution (5242001700FA).

\section{References}

[1] Wang Yong. "Analysis of Transmission Line Protection and Management Based on Power Enterprises." East China Science and Technology: Academic Edition 11 (2014): 233-233.

[2] Wang Tianqiang. "Analysis of the causes and preventive measures against the damage of transmission lines." Collection 36 (2017): 154.

[3] Han Xiaogang. "Analysis of the causes of external damage of transmission lines and preventive measures." Private Technology 11 (2012): 57-57.

[4] Chen Fuzhang; Zeng Xiaofang; Mao Liqin. Analysis of the causes of external force damage on transmission lines and preventive measures. Qinghai Electric Power, 2012, 2.

[5] Guo Shengtian; Hao Yingqin. Analysis of the causes of external force damage on transmission lines and preventive measures. China Electric Power (technical version), 2012, 11: 126.

[6] Ye Shuiyong, Zhang Junlin, Zhang Chi et al. Research and application of intelligent inspection and navigation 
system for power lines. Power and Energy, 2018, 39.1: 24-26.

[7] Zhang Wenfeng Peng Xiangyang Zhong Qing, et al. et al. "Remote Sensing Based on Power Line Safety Inspection Technology and Its Status Quo Outlook." Guangdong Electric Power 27.2 (2014): 1-6.

[8] Xiang Min. Operation and Maintenance of Power Communication Optical Cables in Mountain Areas [J]. Silicon Valley, 2014, 7 (22): 128-129.

[9] Liu Zhaohui. "Remote Sensing Based on Power Line Safety Inspection Technology Status and Prospects." Electronic Production 18 (2017): 32-35.

[10] Xinming T , Fen H U . Development Status and Trend of Satellite Mapping[J]. Spacecraft Recovery \& Remote Sensing, 2018.

[11] Liu Chunxiao. Land use classification in Hedong area of Gansu based on time series MODIS data [D]. Gansu Agricultural University, 2018.

[12] Li Chaoyang. Research on feature extraction and elevation calculation of high-voltage line corridor. Diss. Beijing University of Posts and Telecommunications, 2006.

[13] Peng Xiangyang, Liu Zhengjun, Mai Xiaoming, et al. Safety inspection system and key technology of UAV power line[J]. Remote Sensing Information, 2015 (1): 51-57.

[14] .Feng L, Lin L I . Evaluation of information acquisition capability of optical remote sensing satellites[J]. Optics \& Precision Engineering, 2017, 25(9):2454-2460.

[15] Mu Chao. Research on feature extraction method of power line corridor based on multiple remote sensing data [D]. Wuhan: Wuhan University, 2010.

[16] Wei Xin. Application of Satellite Remote Sensing Image Technology in Electric Power Survey [J]. Collection, 2018, 1: 037 ..

[17] Wenjuan Q I , Xiaomei Y . Mountain and Plain Vegetation Boundaries Extraction in Duchang County Province Jiangxi[J]. Journal of Geo-Information Science, 2017.

[18] Yang Yanjun, Tian Qingjiu, Wang Lei, et al. Crop classification based on GF-1/WFV NDVI time series data [J]. Transactions of the Chinese Society of Agricultural Engineering, 2015, 31(24).

[19] Huang Siyu, Chen Shuisen, Li Dan, et al. Remote Sensing Method Based on Multi-temporal NDVI Phenological Characters for Winter Potato Planting Area in Souith China, Tropical geography, 2016,36(06):976-984.

[20] Jing-Yi Q , Feng-Ling Z, ASIYAManlike, et al. Study on Alfalfa Classification Based on Decision-making Tree and Multiphase Remote Sensing Data[J]. Grass-Feeding Livestock, 2018.

[21] Jakubauskas, Mark E., David R. Legates, and Jude H. Kastens. "Harmonic analysis of time-series AVHRR NDVI data." Photogrammetric engineering and remote sensing 67.4 (2001): 461-470.

Liu Xuying, Guan Yanning, Guo Shan, et al. "Response to vegetation distribution and water level change of Poyang Lake wetland based on time series harmonic analysis." Lake Science 201601 (2016): 195-206.

[22] Zhou Yujie, Wang Juanle, Guo Haihui. Extraction of Cultivated Land Information Based on Harmonic Analysis and Linear Spectral Model[J]. Remote Sensing Technology and Application, 2015, 30(4): 706-713.

[23] He Xiuzhen, Song Naiping, Liu Xiaoyong, et al. Study on the Change Situation of Cultivated Land and Basic Farmland Protection Countermeasures in Yongning County, Ningxia Province[J]. Journal of Agricultural Sciences, 2011, 32(3): 72-76.

[24] Wang Zhihui, Yi Shanzhen. Comparative Study of Different Index Model Methods in Remote Sensing Extraction of Water Body[J]. Science Technology and Engineering, 2007, 7(4): 534-537.

[25] Tao W, Wenhu H, Jing Z, et al. Conservation effectiveness assessment using remote sensing technology in Guizhou Chishui Alsophila National Nature Reserve[J]. Environmental Protection and Technology, 2018. 